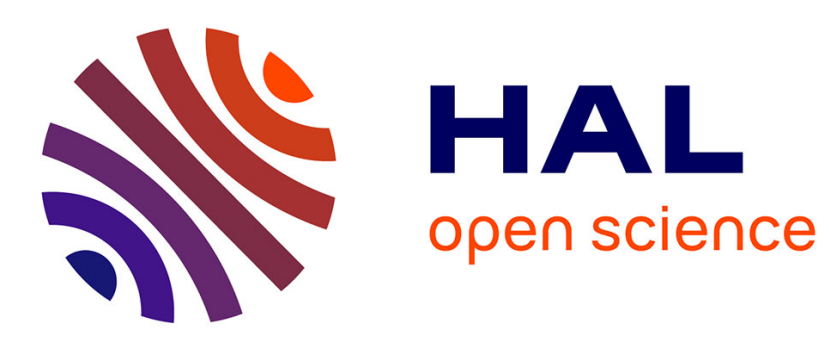

\title{
Derivative Scanning Calorimetry: a new highly sensitive method in differential calorimetry
}

J.-L. Garden, G. Moiroux, C. Dignac, Jacques Chaussy

\section{To cite this version:}

J.-L. Garden, G. Moiroux, C. Dignac, Jacques Chaussy. Derivative Scanning Calorimetry: a new highly sensitive method in differential calorimetry. Thermochimica Acta, 2018, 670, pp.202-210. 10.1016/j.tca.2018.10.020 . hal-01948063

\section{HAL Id: hal-01948063 https://hal.science/hal-01948063}

Submitted on 7 Dec 2018

HAL is a multi-disciplinary open access archive for the deposit and dissemination of scientific research documents, whether they are published or not. The documents may come from teaching and research institutions in France or abroad, or from public or private research centers.
L'archive ouverte pluridisciplinaire HAL, est destinée au dépôt et à la diffusion de documents scientifiques de niveau recherche, publiés ou non, émanant des établissements d'enseignement et de recherche français ou étrangers, des laboratoires publics ou privés. 


\title{
Derivative Scanning Calorimetry: a new highly sensitive method in differential calorimetry
}

\author{
J.-L. Garden ${ }^{*},{ }^{1,2}$ G. Moiroux, ${ }^{1,2}$ C. Dignac,,${ }^{1,2}$ and J. Chaussy ${ }^{1,2}$ \\ ${ }^{1}$ Institut NÉEL, CNRS, 25 avenue des Martyrs, F-38042 Grenoble France \\ ${ }^{2}$ Univ. Grenoble Alpes, Institut NÉEL, F-38042 Grenoble France
}

(Dated: October 17, 2018)

\begin{abstract}
In differential scanning calorimetry, the thermal properties of a sample are recorded as a function of temperature during a predetermined temperature scan. Sometimes, changes of such thermal properties are so fine that it could be interesting to differentiate the recorded signal as a function of time or temperature. This mathematical operation brings out these fine and broad signal variations by removing a 'non-interesting' background. Unfortunately, this also brings out the experimental peak to peak noise, preventing a clear observation. This work presents a new method in differential calorimetry that gives access directly to the temperature derivative of usual differential scanning calorimetry signals. With this method signals are recorded with the same level of noise than those measured by differential scanning calorimetry. As a matter of fact, by means of a mathematical integration, differential scanning calorimetry properties can be recovered with a greater signal to noise ratio. This method can be generalized to any other differential thermal techniques and calorimeters.

PACS numbers: Thermal instruments and apparatus, 07.20.-n, Calorimeters, 07.20.Fw, Specific phase transitions, $64.70 . \mathrm{kj}$, Thermal properties of amorphous solids and glasses: heat capacity, thermal expansion, etc., 65.60.+a, Glasses and polymers, 66.70.Hk,
\end{abstract}

\section{Introduction}

Among experimental methods of measurement, calorimetry has a particular status. It gives access directly to thermodynamic data measured during phase changes or transformations occurring in a given material. Calorimetry was born since the emergence of thermodynamics and the discovery of the fundamental laws driving the evolution of heat and work in a system. By means of experimental thermometry and the measurement of temperature, the notions of specific heat and thermal conductivity have been clarified. Since all materials in every thermodynamic states contain an amount of energy that can be probed, calorimetry covers plenty of different fields of research in science and industry. Despite its universal nature, calorimetry suffers from a lack of selectivity, and it is generally unsuitable for probing the matter at the atomic or molecular scale. In order to avoid this drawback, new sensitive calorimeric methods, such as nanocalorimetry or dynamic calorimetry (ac-calorimetry, temperature modulated calorimetry, ... ) have emerged, allowing a separation between different thermal events involved in a process [1]. Analytical calorimetry is nowadays commonly used in chemistry, physics and biology, as well as in different industries (food, pharmacy, agriculture,... ) [2]. Despite this universal feature, calorimetry still suffers from a lack of resolution, and detecting small thermal events remains difficult, particularly if one want to probe small-scale systems or biological molecules

\footnotetext{
* Corresponding author
}

highly diluted in a solvent. This is because temperature measurement not only gives access to thermal properties of the system under investigation, but also to the thermal properties of the sample-holder, the thermometer itself, the heater, and all the components surrounding the sample (addenda). To avoid this problem, a big forward-step was made by means of differential temperature measurement. With this technique, the differential temperature between a sample and a neutral reference is recorded at any time, like in differential thermal analysis (DTA), differential scanning calorimetry (DSC), differential thermal gravimetry (DTG), and so on. Since differential signals are smaller than direct ones, they must be amplified with low noise amplifiers, increasing the signal to noise ratio. Among differential calorimetric techniques, DSC is certainly the most common $[3,4]$. Using thermopiles in opposition, or by means of two thermometers mounted in differential mode (e.g. Wheatstone bridge), the amount of heat coming from the sample is compared at any time to that coming from a neutral reference whose properties do not change appreciably on the considered temperature interval.

Here we present a new highly sensitive method of measurement in the field of differential calorimetry. This method allows to access directly to fine thermal events that are generally hidden in the signal to noise ratio of classical DSC-calorimeter. This method, called derivative scanning calorimetry (DeSC) may be set up easily on classical DSC-apparatus. In the first part of the paper, we recall the basic principles of DSC measurement, and then we describe the DeSC method. In the second part of the paper, we present experimental data in comparing DSC and DeSC methods on different samples. All the important experimental parameters are examined in 
detail, and an analysis is made on the improvement of the signal to noise ratio with DeSC. In the last parts of the paper, we discuss on the generalization of the DeSC method to other calorimetric methods and give exemple on temperature modulated techniques.

\section{Theory}

\section{Differential scanning calorimetry (DSC)}

A differential scanning calorimeter is merely constituted by two cells or containers, each of them being in good thermal contact with a thermometer. The whole is connected to a heat bath (oven) that controls the temperatures of the two cells. The theory of DSC is well described in benchmark books [3, 4], but for the sake of clarity we briefly recall the basis of the theory. In Fig.(1) a typical differential scanning calorimeter is schematized by thermal conductances and heat capacities. The two cells are assumed to be isothermal, i.e. we do not take into account the possible temperature gradients inside each cell. One cell having the index $S$ contains the sample. Its heat capacity is noted $C_{S}$ and its temperature is noted $T_{S}$. The other cell contains a neutral reference and all the variables have the index $R$. The two cells of the DSC are thermally coupled to the surrounding by the thermal links represented by the conductances $K_{S}$ and $K_{R}$ respectively. These thermal links also account for radiative and convective thermal exchanges with the surrounding. The temperature of the surrounding is noted $T_{0}$. It may be represented by the oven in a heat-flux DSC, for example. This temperature $T_{0}$ is enslaved to follow a scanning temperature ramp (cooling or heating) during an experiment. The thermal links $K_{S}$ and $K_{R}$ allow to the heat to flow between the oven and the cells, whereas the link $K_{\text {int }}$ represents the possible thermal connection between the two cells. Finally, sometimes it may have

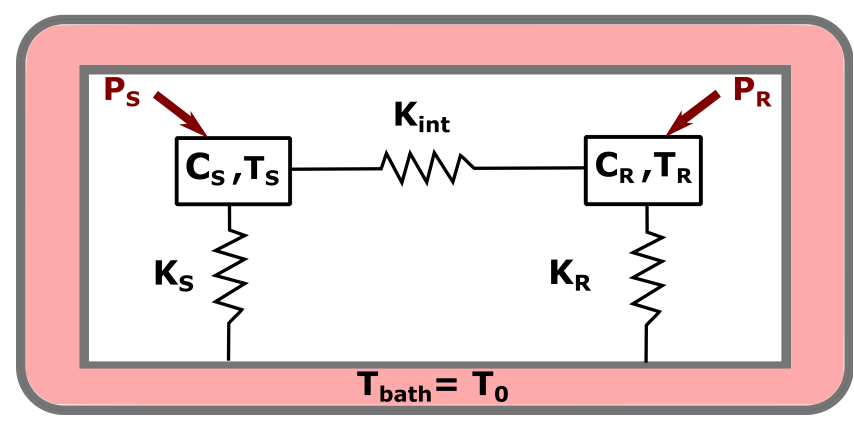

FIG. 1: Thermal scheme of a differential scanning calorimeter represented by thermal conductances, heat capacities and temperatures. a thermal power represented by $P_{S}$ and $P_{R}$ in Fig.(1), that can be generated by heaters directly at the level of the two cells $S$ and $R$ respectively. In power compensated DSC these thermal powers compensate the possible thermal events involved in the sample during a scan. The differential temperature remains equal to zero in this case. In Fig.(1), the temperatures of the cells obey to the following system of differential equations:

$$
\begin{aligned}
& P_{S}=C_{S} \frac{d T_{S}}{d t}+K_{S}\left(T_{S}-T_{0}\right)+K_{i n t}\left(T_{S}-T_{R}\right) \\
& P_{R}=C_{R} \frac{d T_{R}}{d t}+K_{R}\left(T_{R}-T_{0}\right)+K_{i n t}\left(T_{R}-T_{S}\right)
\end{aligned}
$$

It is convenient to write $\Delta T=T_{S}-T_{R} ; \Delta C=C_{S}-C_{R}$; $\Delta P=P_{S}-P_{R} ; \Delta K=K_{S}-K_{R} ; \beta=d T_{R} / d t$.

With these notations, Eq.(1a) becomes:

$P_{S}=C_{S} \times \beta+C_{S} \frac{d \Delta T}{d t}+K_{S} \Delta T+K_{S}\left(T_{R}-T_{0}\right)+K_{i n t} \Delta T$

In making the difference between this latter equation and Eq.(1b), we obtain:

$$
\begin{array}{r}
\Delta P=P_{S}-P_{R}=\Delta C \times \beta+C_{S} \frac{d \Delta T}{d t}+\left(K_{S}+2 K_{i n t}\right) \Delta T \\
+\Delta K\left(T_{R}-T_{0}\right)
\end{array}
$$

Generally, instruments are designed for having $K_{\text {int }}<<$ $K_{S}, K_{R}$. Moreover, if the thermal symmetry of the calorimeter is correct, it could be assumed to a first order that $K_{S} \sim K_{R}$. Under these circumstances, the simplified Eq.(3) is written:

$$
\Delta P=P_{S}-P_{R}=\Delta C \times \beta+C_{S} \frac{d \Delta T}{d t}+K_{S} \Delta T
$$

For heat-flux DSC, the ramp is generated via the oven (thermal surroundings of the cells), and there is no thermal power generated at the level of the two cells $\left(P_{S}=P_{R}=0\right)$. In this case Eq.(4) becomes:

$$
C_{S} \frac{d \Delta T}{d t}+K_{S} \Delta T=-\Delta C \times \beta
$$

This is the basis equation driving a heat-flux DSC. This is a first order linear differential equation for the differential temperature $\Delta T$. During the temperature ramp, all the events occurring in the sample induce a variation of $\Delta C$ which in turn yields to a $\Delta T$ change. When the differential temperature is measured by means of two thermopiles in opposition, it is usual to calibrate them by generating a thermal power $P_{0}$ of given value in one of the cell (with a joule effect heater placed in the sample cell for example). In this case, in stationary conditions we have $\Delta P=P_{0}=K_{S} \Delta T$. The heat exchange coefficient $K_{S}$ is thus measured, knowing that the Seebeck 
coefficient $S$ of the thermopiles has been previously calibrated with two thermometers $S=\Delta V / \Delta T(\Delta V$ is the voltage measured with the thermopiles in opposition). As a consequence, a calibration curve $K_{S}=S \times \Delta P / \Delta V$ is obtained as a function of temperature. This $K_{S}=f(T)$ calibration curve is used to transform the measurand $\Delta T$ in differential heat flux $\Delta P$. The differential Eq.(5) for $\Delta T$ is transformed in the differential equation for $\Delta P$ :

$$
\tau \frac{d \Delta P}{d t}+\Delta P=-\Delta C \times \beta
$$

This equation is of practical importance because experimental data are generally presented by means of graphs $\Delta P=f(T)$ or $\Delta P=f(t)$. With this type of representation, the energy variation involved in the sample during a thermal event is simply obtained by time or temperature integration of the previous curves. The relaxation time $\tau=C_{S} / K_{S}$ is of very high importance in calorimetry because it defines the adiabaticity conditions of the measurement. It governs the power of resolution in temperature (the ability to separate two distinct thermal events for a given value of the temperature rate $\beta$ ). For a given temperature rate $\beta$, when a thermal event generates a power which does not vary too rapidly, we can neglect the derivative in Eq.(6). This yields to a simplified equation for heat-flux DSC:

$$
\Delta P=-\Delta C \times \beta
$$

The measured differential heat flux is in this case directly proportional to the differential heat capacity. Therefore, when $\Delta C$ is changing due to transformations occurring inside the sample during the scans, the signal $\Delta P$ reflects directly this thermodynamic transformations. It is important to have $\beta=\beta_{0}=$ constant in order that the signal does not also reflect the unphysical variation of the temperature scanning rate.

\section{Derivative scanning calorimetry (DeSC)}

As in the previous paragraph, the DeSC method is explained with the help of Fig.(2). In DeSC method, two samples, the most identical as possible (same mass and composition), are placed one in the sample-cell, and the other in the reference-cell of a DSC. Under these circumstances, if we proceed to a classical DSC experiment, the signal will remain equal to zero during the ramp since the same thermal event should occur at the same time, or same temperature, in the two cells (at least to a first order). It is assumed that the two samples are perfectly identical in mass, in composition, and placed in the same way inside the DSC-cells. In a second step, we unbalance the DSC in generating a given temperature difference $\Delta T_{0}$ between the two cells. For that purpose, a supplementary determined power is generated in one of the two cells. In this case, one sample

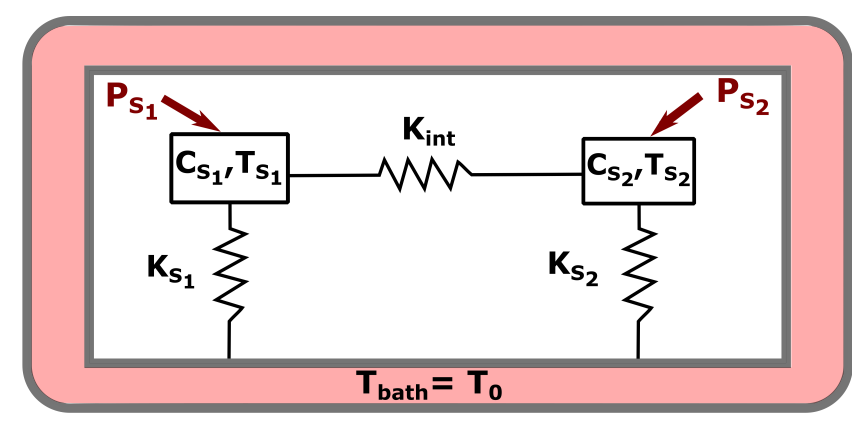

FIG. 2: Thermal scheme of a derivative scanning calorimeter represented by thermal conductances, heat capacities and temperatures.

(that of the sample-cell for example) has a higher temperature than the other one (in the reference-cell). Upon this disequilibrium condition, a classical DSC experiment is then carried out, in starting a temperature scan. Under these experimental conditions, we will show that the measured differential temperature signal is directly proportional to the temperature derivative of the classical DSC signal. With DeSC method, temperature derivative of usual DSC signals are directly recorded. This could be of interest under certain situations, for example when fine thermal events are hidden inside a non relevant constant background. Moreover, this derivative is measured with the same level of noise than in DSC experiment. As a consequence, by means of a mathematical integration the corresponding DSC signal may be recovered with a better signal to noise ratio than usually. Let be $C_{S_{1}}$ the sample-cell heat capacity with the sample and addenda. Let be $T_{S_{1}}$ its temperature. Let be $C_{S_{2}}$ the reference-cell heat capacity with the second sample and addenda. Let be $T_{S_{2}}$ its temperature. Let us note $\Delta C=C_{S_{1}}-C_{S_{2}}$. Let us note $T_{S_{1}}-T_{S_{2}}=\Delta T_{0}+\delta T$ with $\Delta T_{0}$ the temperature disequilibrium imposed by the experimentalist before the experiment, and $\delta T$ the small temperature difference due to potential thermal events appearing inside the samples during the experiment. Like for DSC, we suppose $K_{S_{1}}=K_{S_{2}}=K$. For the sake of simplicity, let us suppose that the temperature disequilibrium has been generated via a heater in the sample cell, in order to have $\Delta P=P_{S_{1}}-P_{S_{2}}=P_{S_{1}}=P_{0}=K \Delta T_{0}=$ constant. Finally, like in DSC, to a first order we suppose $K_{\text {int }}<<K$. With all these new experimental conditions and notations, the system of differential equation (1a and 1b) that governs the temperature of each cell becomes:

$$
\begin{aligned}
P_{0} & =C_{S_{1}} \frac{d T_{S_{1}}}{d t}+K\left(T_{S_{1}}-T_{0}\right)+K_{i n t}\left(T_{S_{1}}-T_{S_{2}}\right) \\
0 & =C_{S_{2}} \frac{d T_{S_{2}}}{d t}+K\left(T_{S_{2}}-T_{0}\right)+K_{i n t}\left(T_{S_{2}}-T_{S_{1}}\right)
\end{aligned}
$$


The analogous of Eq.(4) above becomes:

$$
\Delta P=P_{0}=\Delta C \times \beta+C_{S_{1}} \frac{d \Delta T}{d t}+K \Delta T=K \Delta T_{0}
$$

Since $\Delta T_{0}$ is the predetermined constant part of $\Delta T=$ $\Delta T_{0}+\delta T$, then (9) is transformed into:

$$
C_{S_{1}} \frac{d \delta T}{d t}+K \delta T=-\Delta C \times \beta
$$

which has the same meaning than Eq.(5) of DSC, but this time $\delta T$ is recorded around the constant term $\Delta T_{0}$. This $\delta T$ is due to the evolution along the scan of the differential heat capacity $\Delta C$. It is straightforward to notice that, this time, the differential heat capacity is not the difference between a sample and a neutral reference, but between two same samples having different temperatures:

$$
\begin{gathered}
\Delta C=C_{S_{1}}\left(T_{S_{1}}\right)-C_{S_{2}}\left(T_{S_{2}}\right)=C_{S_{1}}\left(T_{S_{2}}+\Delta T_{0}+\delta T\right) \\
-\left.C_{S_{2}}\left(T_{S_{2}}\right) \simeq \frac{d C_{S}}{d T}\right|_{T=T_{S_{2}}} \times\left(\Delta T_{0}+\delta T\right)
\end{gathered}
$$

Eq.(11) is no more no less the mathematical expression of the temperature derivative of the sample heat capacity. It has been assumed that $C_{S_{1}} \sim C_{S_{2}}=C_{S}$ up to a first order. Indeed, small differences in masses or in compositions of the two samples, as well as thermal contacts, are terms of second order as we will see in the next section. When we want to recover the classical signals of DSC from DeSC, we have to know exactly during the experiment the value of the differential temperature $\Delta T_{0}+\delta T$. In most of the case, we have $\Delta T_{0} \gg \delta T$ because $\delta T$ is the temperature change coming from the fact that a thermal event occurs in one sample just before it occurs in the second one, and the energy difference involved is small. $\Delta T_{0}$ is the initial temperature difference that has been managed by the experimentalist and it is not due to the sample transformations. As a matter of fact, it can be chosen by the experimentalist so as to fulfill the inequality above. Consequently, up to a first approximation the temperature derivative of the heat capacity is simply obtained in dividing the measured differential heat capacity by the initial disequilibrium temperature difference:

$$
\left.\frac{\Delta C}{\Delta T_{0}} \simeq \frac{d C_{S}}{d T}\right|_{T=T_{S_{2}}}
$$

For a heat-flux DSC with a thermopile that has been calibrated, we have $\Delta P=K \Delta T$ with $\Delta P=\Delta P_{0}+\delta P$ with $\Delta T_{0}=P_{0}=K \Delta T_{0}$ and $\delta P=K \delta T$. Eq.(10) becomes:

$$
\tau \frac{d \delta P}{d t}+\delta P=-\Delta C \times \beta
$$

where $\delta P$ is the heat flux difference measured across the thermopiles around the disequilibrium $\Delta P_{0}$. Here again, this initial disequilibrium is not due to sample thermodynamical transformations. The assumption $\delta T<<\Delta T_{0}$ allows to Eq.(13) taking the interesting shape:

$$
\tau \frac{d \delta P}{d t}+\delta P=-\left.\frac{d C_{S}}{d T}\right|_{T=T_{S_{2}}} \times \Delta T_{0} \times \beta
$$

This equation shows that, with the DeSC method, there is one supplementary control parameter other than the usual scanning temperature rate $\beta$. This is the initial temperature disequilibrium $\Delta T_{0}$ managed by the experimentalist before the scan. This allows one to adapt the amplitude of the measured signal to the physical event or transformation that we want to investigate. But, like in DSC, to increase inconsiderately the value of $\Delta T_{0}$ may reduce the resolving power in temperature of the calorimeter, exactly like to increase inconsiderately the scanning rate may do the same.

\section{Experiments}

\section{DSC measurements}

The polytetrafluoroethylene (PTFE) is a calorimetric standard of polymeric system with two crystalline solidsolid phase transitions around room temperature. These two phase transitions have already been studied in our group by means of DSC, and by ac-calorimetry [5, 6]. It is used here in order to test the DeSC method. In Fig.(3), we firstly present raw data of two DSC measurements during increasing temperature scans performed at temperature rates of $1 \mathrm{~K} / \mathrm{min}$ and $0.5 \mathrm{~K} / \mathrm{min}$ respectively. The DSC-apparatus used is a commercial calorimeter micro-DSCIII from Setaram company. The convention for the presented DSC heat flow data is that exothermal events are directed to the top while endothermal events are directed to the bottom. The PTFE sample is a small cylinder of $134.85 \mathrm{mg}$ weight placed inside the batch cell of the microDSCIII. The reference cell is left empty during the experiment. The y-axis represents the heat flow $\Delta P$ since the micro-DSCIII sensor is constituted by two thermopiles in opposition that have been previously calibrated by means of joule effect heater. The two phase transitions are clearly distinguished, the first one around $293 \mathrm{~K}$, due to a twist of the helicoidal macromolecular chain, and the second one around $303 \mathrm{~K}$, due to small conformational changes along the chain $[5,6]$. In Fig.(3), we may observe the proportionality of the measured signal with the scanning temperature rate amplitude. Data of blue square symbols are obtained for the temperature rate of $0.5 \mathrm{~K} / \mathrm{min}$, and those of red circle symbols are for the temperature rate of $1 \mathrm{~K} / \mathrm{min}$. When dividing these raw data by the corresponding scanning temperature rate, we obtain directly the differential heat capacity of the PTFE sample such as given in Eq.(7) (see 


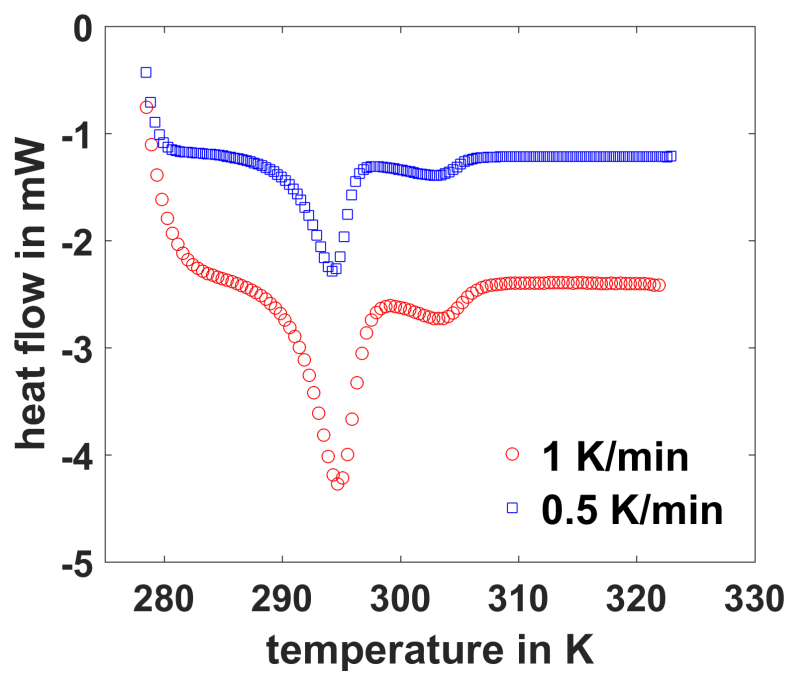

FIG. 3: Heat flow of a PTFE sample measured by DSC at two different heating rates of $0.5 \mathrm{~K} / \mathrm{min}$ (blue square symbols) and $1 \mathrm{~K} / \mathrm{min}$ (red circle symbols).

Fig.(4)). Since the reference cell is empty, the differential heat capacity is equal to the PTFE heat capacity which is presented in Fig.(4) for the two scanning rates. In this figure, the two PTFE heat capacity curves are nevertheless slightly different in particular for the first peak amplitude. This may be due to three different reasons: -it may be due to the calibration of the DSC calorimeter which is dependent on the scanning rates. -it may be due to thermal gradients inside the PTFE sample due to low value of the thermal diffusivity of the PTFE polymer in the substantial volumes used. -it may be due to the intrinsic thermodynamics and kinetics of the PTFE itself, particularly on the first phase transition occurring at around $293 \mathrm{~K} \mathrm{[5].} \mathrm{In} \mathrm{taking} \mathrm{the} \mathrm{time} \mathrm{derivative} \mathrm{of}$ the data in Fig.(3), the more exact Eq.(6) should also be used, but there are no striking differences with $\Delta C$ extracted directly from simpler Eq.(7) such as shown only for scanning rate at $0.5 \mathrm{~K} / \mathrm{min}$ in inset in Fig.(4).

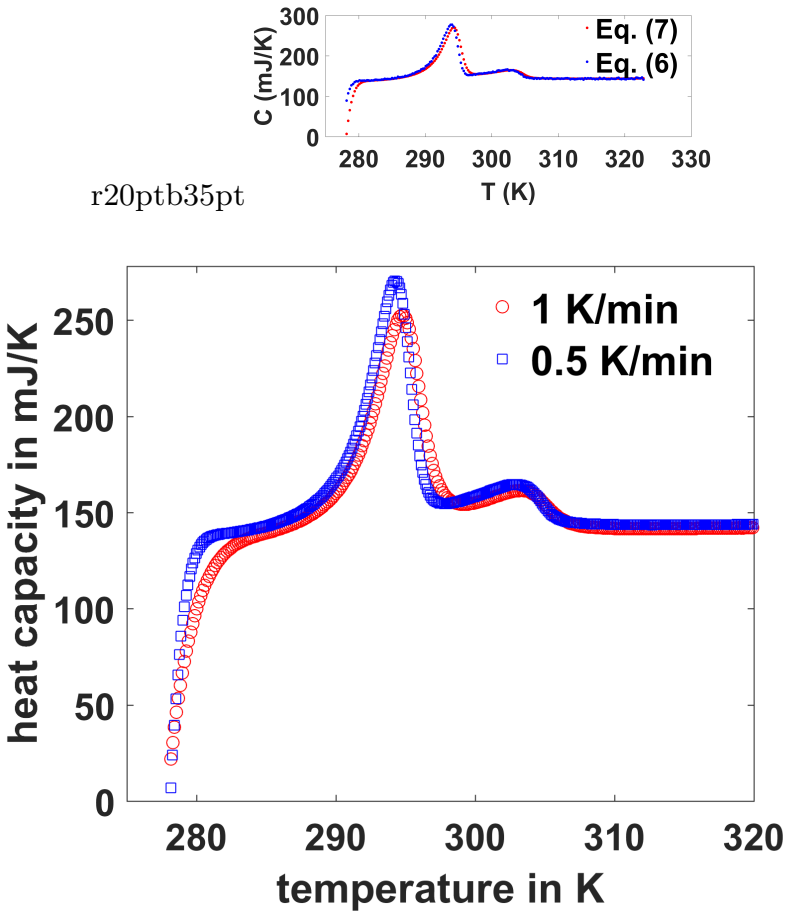

FIG. 4: Heat capacity of a PTFE sample measured by DSC at two different heating rates of $0.5 \mathrm{~K} / \mathrm{min}$ (blue square symbols) and $1 \mathrm{~K} / \mathrm{min}$ (red circle symbols). Inset: Heat capacity of a PTFE sample measured by DSC at the scanning rate of 0.5 $\mathrm{K} / \mathrm{min}$, one using the exact Eq.(6) (blue point symbol) and the other using the simplified Eq.(7) (red point symbol)

\section{DeSC MEASUREMENTS}

\section{Sample and method}

Two PTFE samples have been machined in order to have the same mass. The sample- 1 has a mass of 134.85 $\mathrm{mg}$ (the one used in DSC experiments), while the second one has a mass of $134.82 \mathrm{mg}$. They are both placed in the sample-cell and reference-cell of the micro-DSCIII. In order to generate a temperature disequilibrium between the two cells, the classical batch cells of the micro-DSCIII have been modified. We have included a small heating element in each cell. These small heaters have been glued at the bottom inside the cells. The thin copper connecting wires get out by a small hole at the bottom of each cell and they are sticked inside a thin groove machined along the side of each cell such as depicted in Fig.(5). The thin copper wires then go through the different shields of the calorimeter across small holes. Outside the calorimeter, they are connected to a low noise current source that may generate currents of different amplitudes. From these currents, heat flux disequilibrium (i.e. temperature disequilibrium) of different magnitudes may be generated between each cell. Here, we have supplied power of about 


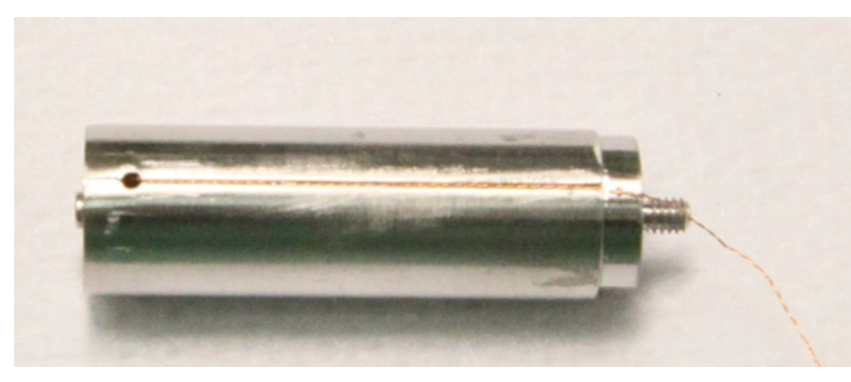

FIG. 5: Modified batch cell of a Setaram microDSCIII calorimeter. The connecting wires get out by a small hole and they are glued inside a thin groove machined along the side of the cell.

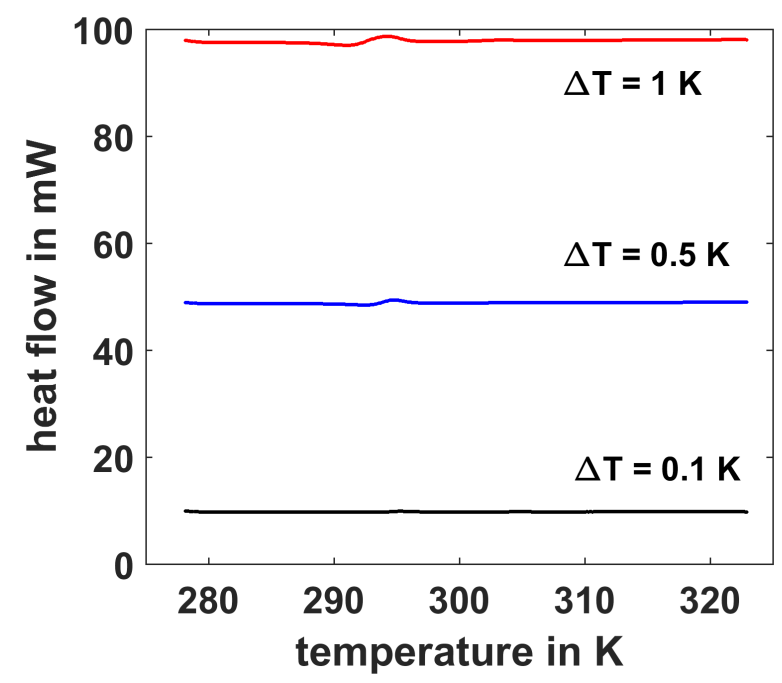

FIG. 6: Full scale DeSC raw data obtained for disequilibrium conditions of: $100 \mathrm{~mW}$ (1K temperature disequilibrium; red data symbols), $50 \mathrm{~mW}$ (0.5 K temperature disequilibrium; blue data symbols), $10 \mathrm{~mW}$ (0.1 K temperature disequilibrium, black data symbols).

$10 \mathrm{~mW}, 50 \mathrm{~mW}$ and $100 \mathrm{~mW}$ respectively, that correspond to temperature differences of about $0.1 \mathrm{~K}, 0.5 \mathrm{~K}$ and $1 \mathrm{~K}$ respectively.

\section{DeSC data}

In Fig.(6), DeSC raw data are presented. These three curves have been obtained for the above different disequilibrium conditions $(0.1 \mathrm{~K}, 0.5 \mathrm{~K}$ and $1 \mathrm{~K})$ with the same increasing temperature rate of $0.5 \mathrm{~K} / \mathrm{min}$. As can be seen for the three conditions, there is a varying signal around the initial disequilibrium value such as expected. How to treat these data is discussed in a next section.
Influence of the disequilibrium

For the sake of clarity, in order to emphasize the variations of each signal, in Fig. (7) we have subtracted for each of them a value close to the disequilibrium power, and we have shown them on the same graph. The low temperature baseline is now close to zero for all the curves. A first remark that can be made is that, now, the two PTFE phase transitions appear as a double peak for the first one (a first downward peak followed by an upward one), and an upward smaller peak for the second phase transition. One can point out that, the higher the initial disequilibrium is, the higher the signal variation is with amplitudes of the peaks becoming more and more pronounced (red circle symbols for $1 \mathrm{~K}$ disequilibrium, blue square symbols for $0.5 \mathrm{~K}$ disequilibrium, black triangle symbols for $0.1 \mathrm{~K}$ disequilibrium). In other word, the higher the temperature difference between the two samples during a scan, the higher the energy involved in the measurement. This proves that this initial temperature difference is a new interesting control parameter which can be tuned in order to increase the signal intensity during a scan. Obviously, to increase inconsiderately this initial disequilibrium leads to a decrease of the resolving power of the instrument. The resolving power of the calorimeter is the ability of the instrument to separate two close thermal events in a given sample for a given scanning rate. We may easily understand that if the temperature difference is higher, or in the same order of magnitude, than the temperature difference between the two events that we want to separate, the temperature derivative of such events cannot be correctly recorded anymore. In this case, two separated heat capacity peaks may lead to a broad asymmetrical peak. In Fig.(7), this later effect starts to be observed for the higher temperature difference of $1 \mathrm{~K}$ (red circle symbols). The thermal events occur on a larger temperature interval than for the experiments with smaller temperature differences. This is exactly the same effect that occurs in DSC when the scanning rate becomes too high with respect to the thermal time constant of the calorimeter. As a conclusion, the experimentalist has to adapt this new tuning parameter to the physics of the process under study, exactly like he has to adapt the scanning rate. Finally, as can be estimated from the three graphs, the assumption discussed beforehand that the small measured temperature difference $\delta T$ is negligible as compared to the imposed temperature difference $\Delta T_{0}$ is fulfilled. To prove it, we have $\delta T / \Delta T_{0}=\delta P / \Delta P_{0} \sim 2 \%$ for the three experiments (where $\delta P$ is the maximum heat flux difference taken between the maximum and the minimum of the peaks of the $293 \mathrm{~K}$ phase transition). 


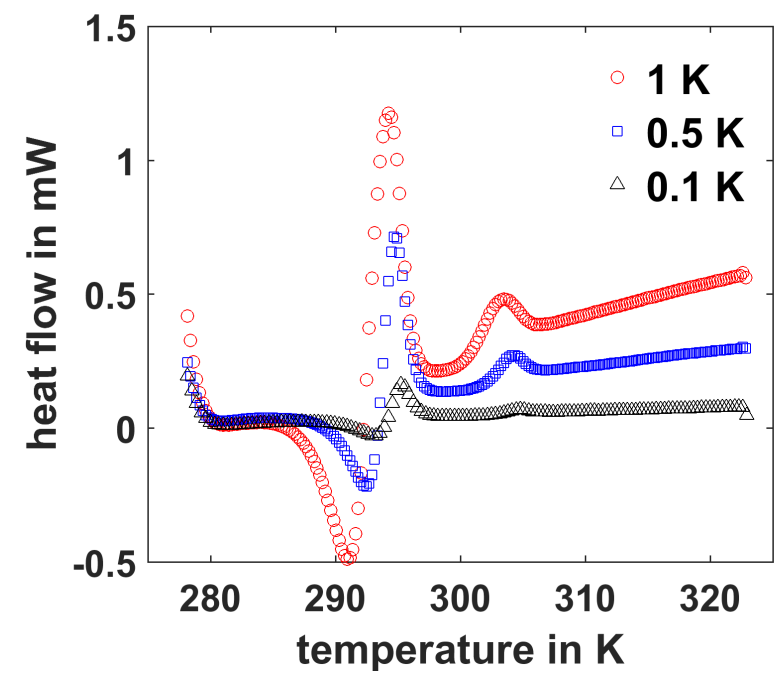

FIG. 7: Rescaled DeSC data where each heat flow disequilibrium have been subtracted to raw data. Disequilibrium of $100 \mathrm{~mW}$ corresponds to red circle symbols, of $50 \mathrm{~mW}$ to blue square symbols, of $10 \mathrm{~mW}$ to black triangle symbols respectively. The higher the initial disequilibrium is, the higher the heat flow signature of the two PTFE phase transitions is, but the larger the temperature transition range is.

\section{Influence of the scanning rate}

In the following Fig.(8), we compare two DeSC measurements carried out with the same initial temperature difference $\Delta T_{0}=0.5 \mathrm{~K}\left(\Delta P_{0}=50 \mathrm{~mW}\right)$, but at two different scanning rates (blue square symbols for $0.5 \mathrm{~K} / \mathrm{min}$ and red circle symbols for $1 \mathrm{~K} / \mathrm{min}$ ). As can be observed, the higher the scanning rate is, the higher the signal variation is, like in DSC. We also observe that the temperature range over which the phase transition takes place is higher for the higher scanning rate. This confirms another time that, the higher the intensity of the tuning parameter, the smaller the resolving power of the measurement.

\section{Noise reduction in DeSC method}

Since the DeSC method gives rise to a direct access to the temperature derivative of usual DSC signals, it may be interesting to recover such DSC signals. We present here a first simple data treatment for that purpose. But, we would like to show here that it is possible to recover such signals with a higher signal to noise ratio. From the raw data of the DeSC measurement with $\Delta T_{0}=0.5 \mathrm{~K}$ and $\beta=0.5 \mathrm{~K} / \mathrm{min}$ (see the square blue symbols curve in Fig.(8) or the middle blue curve in Fig.(6)), we adjust a sigmoid to the DeSC curve that is then subtracted in order to obtain two flat baselines equal to zero in the

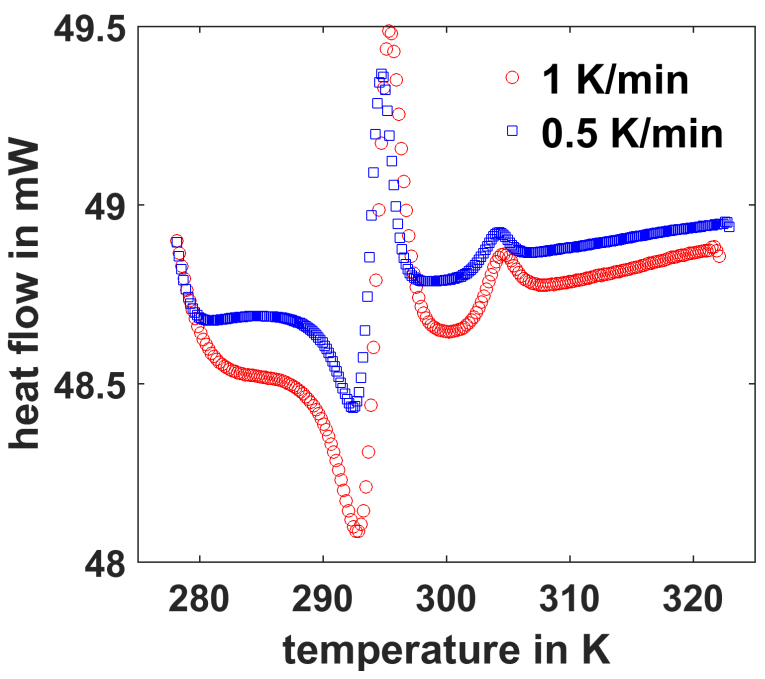

FIG. 8: Two raw DeSC data obtained for a same initial disequilibrium of $50 \mathrm{~mW}$ (corresponding to a temperature disequilibrium of $0.5 \mathrm{~K}$ ) at two different temperature rates of 0.5 $\mathrm{K} / \mathrm{min}$ (blue square symbols) and $1 \mathrm{~K} / \mathrm{min}$ (red circle symbols). The higher the scanning rate, the higher the signal variation.

low and high temperature ranges outside the transitions range. The result of such correction is shown in Fig.(9) (see the red point symbol curve) along with the temperature derivative of the DSC curve that have been obtained at the same rate of $\beta=0.5 \mathrm{~K} / \mathrm{min}$ (square blue symbols DSC curve in Fig.(3)). The result of such mathematical differentiation is shown on the same scale than for the corrected DeSC curve in Fig.(9) (see the blue cross symbol curve in Fig.(9)). As can be observed, the two graphs in Fig. (9) are quite similar, confirming that what is measured by DeSC is the temperature derivative of classical DSC signals. A magnification of the two previous curves in the flat part of the high temperature regime (see inset in Fig.(9) between $310 \mathrm{~K}$ and $320 \mathrm{~K}$ ) shows that the short term noise is lower on the DeSC signal than on the mathematically differentiated DSC signal. It is obvious that in integrating the two curves, we will obtain a DSC signal (obtained from DeSC) with less noise than classical one (obtained from DSC). As a conclusion, the DeSC method allows to recover DSC signals with less noise than if there are directly measured by DSC. This could be very important in many different applications. For example this could be interesting in the case of highly diluted biological macromolecules thermal denaturation in solution, where the associated heat capacity peak is generally small and hidden in an non-interesting high heat capacity background coming principally from the aqueous solvent. 

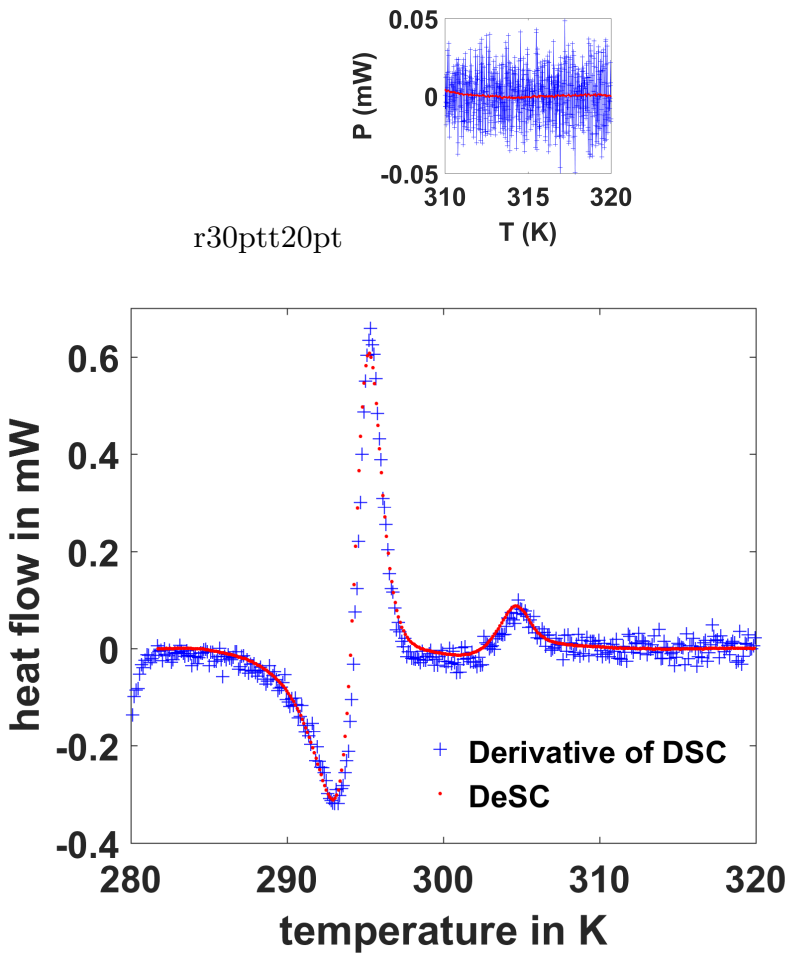

FIG. 9: Two heat flow signals are presented on the same scale, one coming from data processing of DeSC experiment (red point symbols), and the other coming from mathematical differentiation of corrected DSC signal (blue cross symbols). The comparison of the two signals confirms that with DeSC method we access to a temperature derivative of DSC signal. Inset: magnification of the two signals in the flat part of the high temperature range between $310 \mathrm{~K}$ and $320 \mathrm{~K}$. Short term noise is smaller with DeSC than with DSC.

\section{Influence of the differential mass}

When the masses of the two samples are substantially different, this may introduce a supplementary parasitic temperature difference during the scan. In dividing the differential heat capacity by the PTFE mass in Fig.(4), the PTFE specific heat is obtained as a function of temperature. From this measurement, the PTFE specific heat is directly obtained because the reference-cell is empty. The difference in the masses of the two samples for DeSC measurement is of $30 \mu \mathrm{g}$ only. This is merely the mass inaccuracy given by our balance. Such mass difference may induce a supplementary heat flux disequilibrium equal to $\Delta m \times c_{P T F E} \times \beta$ during the scan made at the temperature rate $\beta$. This is a supplementary contribution which has to be added to the right-hand-side term of Eq.(13) of DeSC. In this case, the disequilibrium is due to the differential mass and not only to the initial temperature difference. Such parasitic term has to be compared with respect to the principal DeSC term given by $\Delta C \times \beta$. This comparison is done in Fig.(10) where,

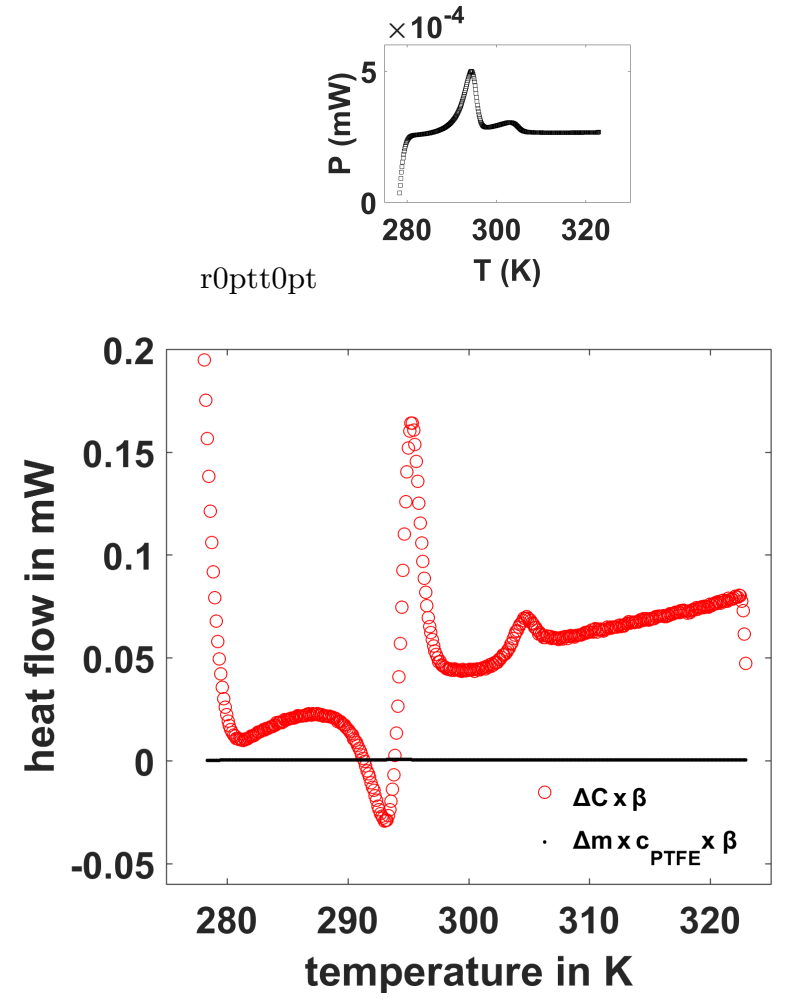

FIG. 10: Two heat flow signals are presented on the same scale $(-0.06$ to $0.2 \mathrm{~mW})$. One is the principal term of a DeSC experiment measured at the scanning rate of $0.5 \mathrm{~K} / \mathrm{min}$ (red circle symbols) where the constant $10 \mathrm{~mW}$ term has been subtracted, and the other is the parasitic DSC term coming from the mass difference between the two samples (black point symbols). The latter is negligible, practically equal to zero on this scale, by comparison with the usual DeSC term. Inset: the same parasitic term due to the differential mass is presented on a lower scale ( 0 to $5.10^{-4} \mathrm{~mW}$ ) showing that its variations are completely negligible compared to the DeSC variations.

with the help of the DSC curve in Fig.(4), we have introduce $\Delta m \times c_{P T F E} \times \beta$ in the DeSC raw data. This was made for the experiment with the temperature difference of $0.1 \mathrm{~K}$ and a scanning rate of $0.5 \mathrm{~K} / \mathrm{min}$ (the lowest signal). In inset in Fig.(10), we present a magnification of this parasitic term which reproduces the PTFE heat capacity such as mentioned. As can be seen, variations in PTFE specific heat give variations in heat flow of about few $10^{-4} \mathrm{~mW}$ for this contribution, which is negligible with respect to the principal DeSC term. This being, we thus have observed that a DeSC experiment can be implemented in a DSC-calorimeter without the use of a supplementary heater. Indeed, let us consider that in one of the cell of the DSC-calorimeter we add a reference material of a given mass with a specific heat which does not vary significantly inside the considered temperature range. For example, let us place a copper piece of mass $m$ with one of the two samples in the calorimeter. Un- 


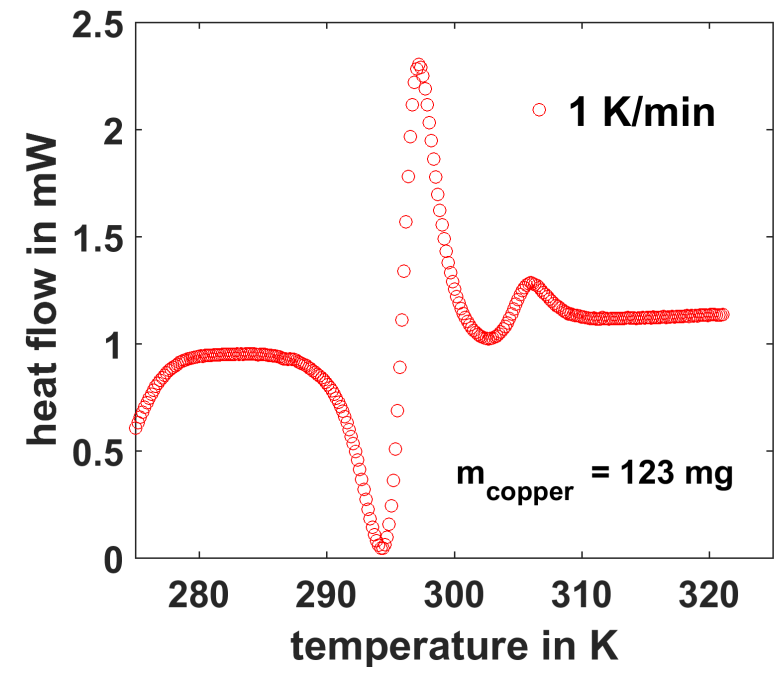

FIG. 11: DeSC raw data carried out under a disequilibrium of about $1 \mathrm{~mW}$ (i.e. temperature disequilibrium of about 10 $\mathrm{mK}$ ) produced by a supplementary mass of copper of $123 \mathrm{mg}$ in one of the cell of the DSC. There is no heat supplied by a heater in this experiment.

der these circumstances, during the scan, there will be a supplementary $\Delta c_{c o p}=m \times c_{c o p}$ term which will induce a quite constant $\Delta T$ term, with $c_{c o p}$, the copper specific heat. For instance, this $\Delta T$ contribution will be as constant as $c_{c o p}$ on all the temperature range. This $\Delta T$ naturally will unbalance the calorimeter, making possible to carry out a DeSC measurement. This is what we have done in using a supplementary piece of copper of mass of $123 \mathrm{mg}$ placed with one of the two PTFE samples (the PTFE samples used in this try have a mass of about $248.15 \mathrm{mg}$ ). In Fig.(11), we present the DeSC experiment carried out under these conditions at a rate of $1 \mathrm{~K} / \mathrm{min}$. As can be shown, the derivative of the PTFE heat capacity is measured again. It could also be pointed out that a disequilibrium of about $1 \mathrm{~mW}$ is obtained by this means, that corresponds to a temperature disequilibrium of about $10 \mathrm{mK}$. For obtaining greater values of the temperature difference between the two cells (such as $0.5 \mathrm{~K}$ or $1 \mathrm{~K}$ for example like we did with heater), copper masses on the order of grams are necessary.

\section{Derivative Scanning Calorimetry for modulated calorimetry: ac-DeSC}

As a sake of generality, in this section we will show experimentally how the DeSC method is also suitable for any other calorimetric methods, and in particular for temperature modulated calorimetry. The PTFE is a polymer which has been studied in our group by ac-

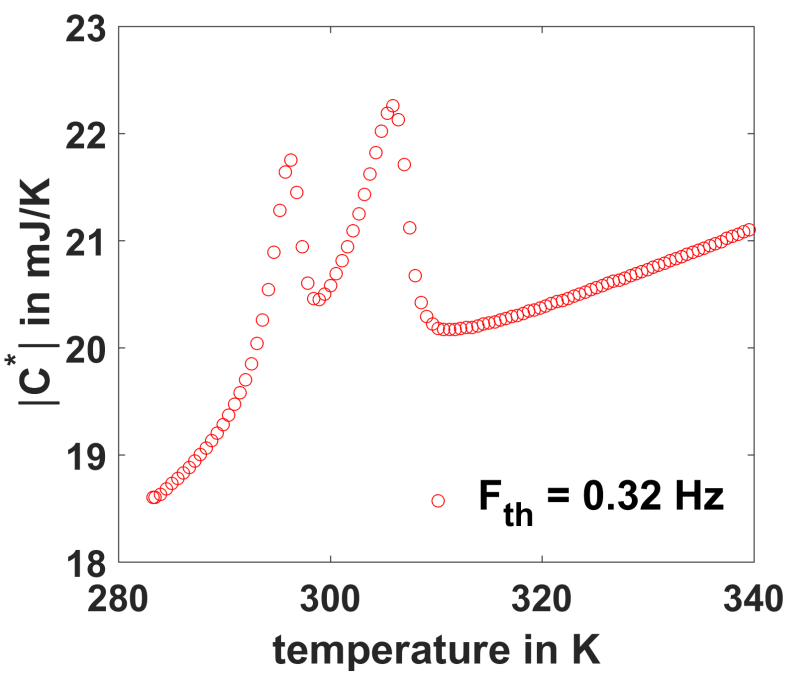

FIG. 12: Modulus of the dynamic heat capacity of a PTFE sample as measured by ac-calorimetry at a thermal frequency of $0.32 \mathrm{~Hz}$. The kinetic effect due to temperature oscillation is clearly observed on the first phase transition around $294 \mathrm{~K}$ if we compare to DSC measurement in Fig.(4).

calorimetry [5-8]. The influence of the oscillating temperature frequency on the recorded dynamic heat capacity for the $294 \mathrm{~K}$ first crystalline phase transition has been investigated [5-8]. The ac-calorimeter used is based on two microfabricated cells described in Ref.[5]. On one cell there is a heater supplying the oscillating power $P_{a c}=P_{0} \cos (\omega t)$ while on the other cell there is a platinum thermometer recording the oscillating temperature $T_{a c}=\delta T_{a c} \cos (\omega t-\varphi)$, the PTFE sample being compressed between such two cells during a measurement ensuring thermal homogeneity. The dynamic heat capacity is obtained by means of the classical expression $C^{*}=\dot{Q}_{a c} / \dot{T}_{a c}=P_{a c} / i \omega T_{a c}$. On the graph in Fig. (12), the modulus of the dynamic heat capacity $\left|C^{*}\right|=P_{0} / \omega \delta T_{a c}$ is represented as a function of temperature during a mean temperature scanning rate of $0.5 \mathrm{~K} / \mathrm{min}$ and an amplitude of temperature oscillation of about $0.1 \mathrm{~K}$ at a thermal frequency of about $0.32 \mathrm{~Hz}$. The kinetic effect occurring on the $294 \mathrm{~K}$ phase transition is clearly revealed (smaller amplitude of the peak) if we compare with the same transition measured by DSC (see Fig.(4)). In order to implement the DeSC method for ac-calorimetry, a microfabricated cell has been developed for that purpose (see the schematic drawing in Fig.(13)). The thermometric cell is now composed of two separated thermometers with two corresponding dc-heaters at the back face of the suspended membrane. One of this heater is used for producing the temperature difference between the two thermometers (named $\Delta T_{d c}$ in Fig.(13)). The other cell contains a heater on all the surface that may supply an oscillating power (named $P_{a c}$ in Fig.(13)) in the sam- 


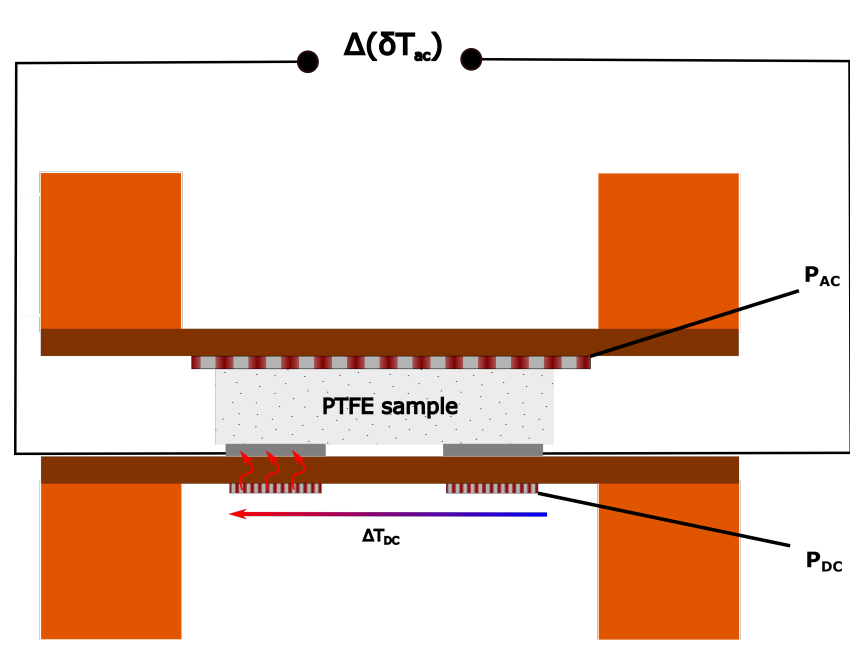

FIG. 13: Schematic drawing showing the principle of functioning of the two microfabricated cells for ac-DeSC. The PTFE sample is enclosed between two suspended membranes one containing the ac-heater and the other one containing two thermometers and two corresponding dc-heaters at the other face of the same membrane. The dc temperature disequilibrium is made by the use of the dc-heaters. Two thermally independent parts of the PTFE sample oscillate at a given frequency due to the ac-heater, each of them being at different dc temperature.

ple and the thermometric cell. The schematic drawing in Fig.(13) shows how oscillating heat is generated in the sensor, and how the oscillatory differential temperature is recorded. Under these circumstances, we have two thermally independent parts of the PTFE sample which oscillate, receiving the same oscillatory power, but each of them being at a different dc temperature. The recording differential ac temperature is just the signature of the differential heat capacity between each part of the PTFE sample being at a different dc temperatures $\Delta \delta T_{a c}=\delta T_{a c}\left(T_{1}\right)-\delta T_{a c}\left(T_{2}\right)=\left(P_{0} / \omega\right) \times \delta(1 / C)$ with $T_{1}=T_{2}+\Delta T_{d c}$. For instance, the differential measurement yields to the difference of the inverse heat capacities $\delta(1 / C)=1 / C_{1}\left(T_{1}\right)-1 / C\left(T_{2}\right)$ of the same material, but taken at different dc temperatures $T_{1}$ and $T_{2}$. These are the experimental requirements for the DeSC method. The derivative of the PTFE sample is just obtained knowing that $\delta(1 / C)=-\delta C / C^{2}$. We have to divide $\delta C=-\left(\omega C^{2} / P_{0}\right) \times \Delta \delta T_{a c}=-\left(C / \delta T_{a c}\right) \times \Delta \delta T_{a c}$ by $\Delta T_{d c}$ to obtain the derivative of the sample heat capacity. The measured differential signal of the two oscillating temperatures $\Delta \delta T_{a c}$ is thus directly proportional to the derivative of the sample heat capacity. The result of such an experiment is depicted in Fig.(14). The curve with blue square symbols is the mathematical temperature derivative of the $\left|C^{*}\right|(T)$ curve in Fig.(12) obtained by classical ac-calorimetry, while the curve with red cir-

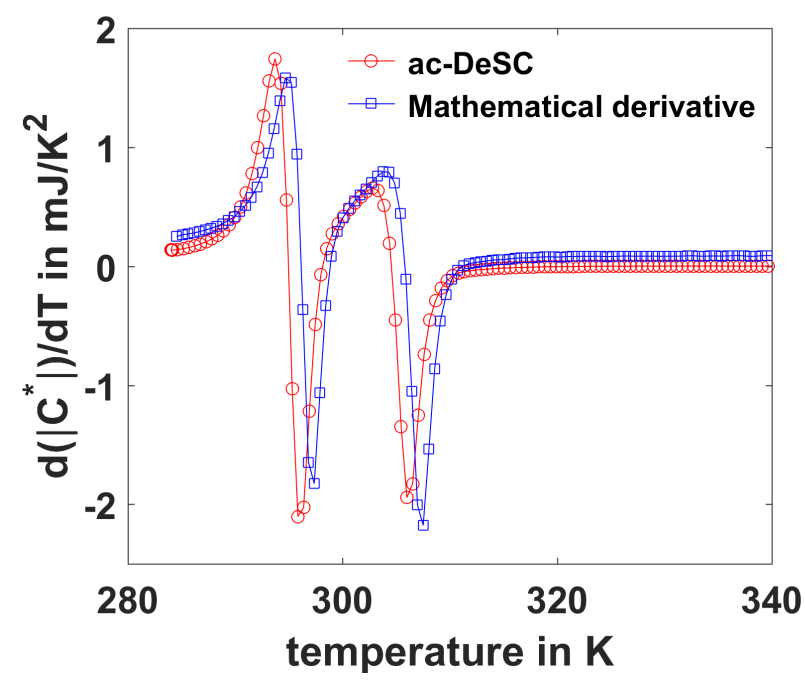

FIG. 14: Two temperature derivatives of the modulus of the dynamic heat capacity of a PTFE sample are presented. The curve with blue square symbols is the mathematical temperature derivative of the curve in Fig.(12). The curve with red circle symbols is obtained by ac-DeSC such as explained in the text.

cle symbols has been obtained from the measured $\Delta \delta T_{a c}$ at the same frequency, the same scanning rate, and with a temperature difference of about $1.3 \mathrm{~K}$ between the two platinum thermometers. This latter signal has been normalized by a coefficient taking into account the amplifier gain and the calibration of the thermometers. The visible drift in temperature between both curves comes from the fact that we have presented the normalized differential signal $\Delta \delta T_{a c}$ has a function of the absolute temperature $T_{1}$ of the coldest thermometer. The generalisation of the DeSC method for all other temperature modulated calorimetric techniques is obvious.

\section{Glass transition measured by DeSC}

The DeSC method may be useful in the study of the glass transition. In most of the cases, during vitrification heat capacity jumps may be of small magnitudes and may take place on broad temperature ranges. In this case, due to the improvement of the signal to noise ratio, DeSC may be useful. In this section, we focalise on DeSC measurements performed on a model of polymeric glass-former, the PolyVinylAcetate [PVAc, $\left(\mathrm{C}_{4} \mathrm{H}_{6} \mathrm{O}_{2}\right)_{n}$ ]. Fig.(15) shows six DeSC curves with heating scans at 0.5 $\mathrm{K} / \mathrm{min}$ and temperature disequilibrium of $1 \mathrm{~K}$. These six curves have been obtained on the same PVAc sample, measured after a cooling rate of $-1.2 \mathrm{~K} / \mathrm{min}$, followed by different aging times at the temperature of $293 \mathrm{~K}$ (the starting temperature is of about $363 \mathrm{~K}$ where the sample 

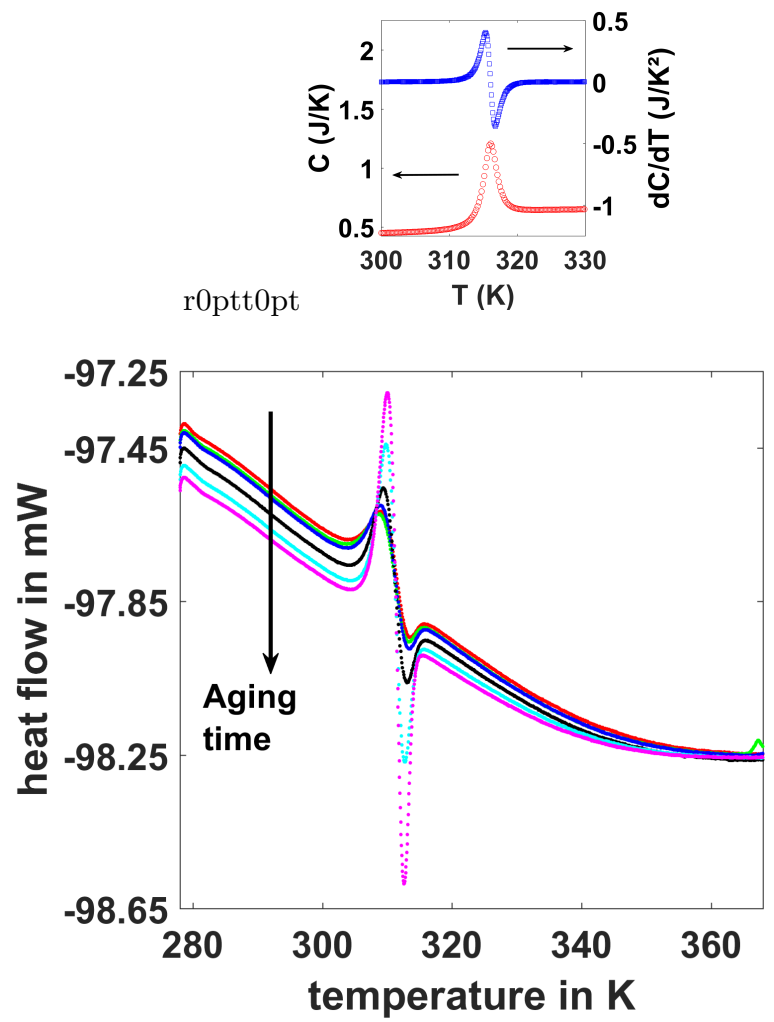

FIG. 15: DeSC raw data of PVAc sample during heating scans at $0.5 \mathrm{~K} / \mathrm{min}$ with temperature disequilibrium of about $1 \mathrm{~K}$ (heat flow disequilibrium of $100 \mathrm{~mW}$ ). The scans are made after cooling from the starting temperature of $363 \mathrm{~K}$ at $1.2 \mathrm{~K} / \mathrm{min}$ followed by an aging process with time periods of $10 \mathrm{~min}, 30 \mathrm{~min}, 100 \mathrm{~min}, 300 \mathrm{~min}, 1000 \mathrm{~min}, 3000 \mathrm{~min}$ respectively, and then followed by a new cooling at $-1.2 \mathrm{~K} / \mathrm{min}$ until the lowest temperature of $278 \mathrm{~K}$ just before the starting of the scanning ramps. The black arrow points out the evolution of the different curves with increasing aging time. Inset: Left-hand scale : DSC heat capacity of PVAc during a heating scan at $1 \mathrm{~K} / \mathrm{min}$ after the same type of cooling than for DeSC experiment with an aging time of $1200 \mathrm{~min}$ at $293 \mathrm{~K}$ (red circle symbols). Right-hand scale: temperature derivative of the DSC heat capacity of PVAc such as measured.

is under the supercooled liquid state). The aging times were of $10 \mathrm{~min}, 30 \mathrm{~min}, 100 \mathrm{~min}, 300 \mathrm{~min}, 1000 \mathrm{~min}$, 3000 min respectively. After this aging process, the two samples have been cooled both toward the lowest temperature of $278 \mathrm{~K}$ before the starting of the heating scans.

As can be seen in Fig.(15), the DeSC signals look like temperature derivatives of classical DSC signals measured by DSC. In inset in Fig.(15), we present a classical DSC signal recorded at $1 \mathrm{~K} / \mathrm{min}$ with the associated mathematical temperature derivative on the same graph (up curve with the right hand scale). This DSC experiment corresponds to a cooling followed by an aging process of $1200 \mathrm{~min}$ at the same aging temperature of 293 $\mathrm{K}$. If we do not consider the decreasing slopes observed on the DeSC curves, they all have the same shape of a DSC temperature derivative such as presented in inset. The two peaks around $313 \mathrm{~K}$ correspond to the derivative of the enthalpy recovery peak usually measured by DSC. It may be pointed out that all the curves are well separated, particularly at low temperatures, which correspond to different temperature variations (slopes) of the heat capacity during heating. These differences are little by little attenuated until around $363 \mathrm{~K}$ at the highest temperature where all the memory of the PVAc sample has been erased. Before this last temperature, the PVAc follows an irreversible path depending on its history, especially due to the different aging times at $293 \mathrm{~K}$. As a conclusion, by means of DeSC it is possible to observe classical events, like changes in the magnitude of the enthalpy recovery peaks as a function of the aging times, like in DSC, but it is also possible to clearly reveal finer events, like changes in the slopes of the $\mathrm{Cp}(\mathrm{T})$ curves during heating, which is generally less accessible by DSC.

\section{Generalisation of DeSC to other thermal analysis techniques}

A generalisation of the method can be made to every other thermal analysis techniques. Let us suppose a physical property $M$ that must be measured. Let us then suppose that the property depends on temperature $M=M(T)$, and that the experimental technique used consists in varying the temperature in order to access to the interesting changes of this property. For example, $M$ could be the mass of a sample in thermogravimetry. If the experimental set-up is differential, i.e. it contains two crucibles which can contain two samples. If the set-up allows the control of the temperature of the two crucibles so that a temperature difference may be generated between them, then the recorded signal of such set-up can yield to the temperature derivative of the given property along the temperature scan. For example, if the property $M=M(T)$ is the sample mass in a differential thermogravimeter, then by putting two identical samples in each crucible, and by producing an initial disequilibrium dc temperature, then the recorded signal gives directly the temperature derivative of the sample mass:

$$
M_{1}\left(T_{1}\right)-\left.M_{2}\left(T_{2}\right) \sim \frac{d M}{d T}\right|_{T} \times \Delta T
$$

Here again, to use directly a derivative as a recording signal may improve the signal to noise ratio as compared to usual differential thermogravimetric techniques. 


\section{Discussion on problems encountered with DeSC}

Besides all advantages of the DeSC technique discussed in the text, we would like to mention in this short discussion some problems we have faced during these first measurements. Although the temperature derivative of classical DSC signals can be directly recorded, with a smaller level of noise than for DSC, it remains rather difficult to recover the exact DSC signal. Indeed, we have to bear in mind that with DeSC all usual second order parasitic effects are also magnified. For example, even if the two samples are the most identical as possible in their compositions, or in their masses, if they are positioned slightly differently in the two crucibles of the DSC (different thermal contacts), this small difference may be amplified by the method itself. It is then difficult afterward to recover a perfect DSC signal. A second point is that the dissymmetry of the calorimeter is also magnified with DeSC. Indeed, this time it unbalances the sample 1 with respect to the sample 2, while usually this dissymmetry unbalances the sample with respect to the reference. This is why we have difficulty to recover a perfect "flat baseline" after integration of the DeSC signal. However, this may be a very efficient method to calibrate the calorimeter. A calorimeter, which may be calibrated taking into account second order dissymmetrical terms, may be more accurate on first order signals. A third point, which is usual in the integration process of differentiated signals, is that we need a supplementary measurement in order to access to the absolute value of the sample heat capacity. We can imagine a first DeSC measurement made with two identical references. Only in a second step, we perform the second measurement with the two samples. Finally, we may subtract the two previous results before the integration in order to recover the classical DSC signal. This two measurement steps have the merit to eliminate the mentioned asymmetry problem. Finally, more numerical data-processing developments may also be necessary to be able to recover the most perfectly as possible classical heat flux signals (heat capacity). As a final point of the discussion, we can ask whether new or additional physical or thermodynamic information can be gained with DeSC method if we compare with DSC. Since DeSC is like a magnification process obtained with a higher signal to noise ratio, it is indeed possible to have access to very tiny thermal events which usually are hidden in the noise of the DSC. By analogy with microscopy, DeSC is like an eyepiece of a microscope with a higher magnification.
We have presented a new technique in the field of differential scanning calorimetry. This new method is able to record directly the temperature derivative of signals (heat flow, heat capacity) which are usually recorded by differential calorimetry techniques. We have discussed the influence of different parameters on this new method. We have given few examples of measurements, and discussed the potentiality of this method in terms of signal to noise ratio. The derivative scanning calorimetry has been generalized to other differential thermal analytical techniques, enlarging the research field in experimental thermal analysis and calorimetry.

\section{acknowledgments}

We would like to thank C. Lavorini for having carried out the first series of DeSC experiments with a modified DSC, and J. Richard for data processing on PVAc. We would also like to thank the technological expertise of the "pôle de capteurs thermométriques et calorimétrie", of the "pôle électronique", and of the "pôle cryogénie" of the laboratory.

[1] I. Hatta, A.J. Ikushima, Jap. J. Appl. Phys. 20 (1981) 1995.

[2] P.K. Gallagher, Handbook of Thermal Analysis and Calorimetry, Elsevier, 1998.

[3] G. Höhne, W. Hemminger, H.-J. Flammersheim, Differential Scanning Calorimetry, An introduction for Practitioners, Springer, 1996.

[4] P. Claudy. Analyse calorimétrique différentielle, Lavoisier, 2005.

[5] J.-L. Garden, J. Richard, H. Guillou, O. Bourgeois, Thermochim. Acta 461 (2007) 122.

[6] J.-L. Garden, H. Guillou, A.F. Lopeandia, J. Richard, J.S. Heron, G.M. Souche, F.R. Ong, B. Vianay, O. Bourgeois, Thermochim. Acta 492 (2009) 16.

[7] E. Château, J.-L. Garden, O. Bourgeois, J. Chaussy, Appl. Phys. Lett. 86 (2005) 151913.

[8] J.-L. Garden, J. Richard, H. Guillou, O. Bourgeois, Advances in Chemistry Research, Nova, 2011, Volume 7, Chapter 7. 